\title{
Optimization of Monolayer Hydrophilic Matrix Tablets Containing Nisoldipine Solid Dispersion Using D-optimal Design
}

\author{
Sandeep Kumar ${ }^{1 *}$, Romi Singh1, Roopnarayan Gupta² \\ ${ }^{1}$ Product Development and Research, Sun Pharmaceutical Industries Limited, Sector-18, Gurugram-122015, INDIA. \\ ${ }^{2}$ Department of Pharmaceutical Sciences and technology, Birla Institute of Technology, Mesra, Ranchi, INDIA.
}

\begin{abstract}
Background: Nisoldipine (NIS) is a BCS-class II drug with a low oral bioavailability ( $5 \%$ ). The popular marketed product is a trilayer Geomatrix ${ }^{\circledR}$ system. Although it provides for drug release control of NIS, a trilayer matrix system is a complex design, which is difficult to manufacture on an industrial scale using conventional press. The present study discusses an alternate approach to improve drug dissolution using solid dispersion (SD) method followed by design of a simple monolayer controlled release (CR) matrix system. Methods: Aqueous drug solubility study was carried out at various copovidone levels. NIS-copovidone SD was prepared by hot-melt extrusion and was characterized by infrared spectroscopy (FTIR) and scanning electron microscopy (SEM). A risk assessment based approach was used to select the factors and levels affecting the design of a monolayer matrix tablet. A custom screening design of experiments was used to screen the type and level of excipients followed by a D-optimal mixture design and the dissolution profile was selected as the main response to statistically analyze the data to optimize the final formula. Results: A solvent free approach by hot-melt extrusion was used to prepare NIS-copovidone solid dispersion at $20 \%$ drug load, which lead to a significant improvement in dissolution $\left(\mathrm{T}_{90}=30 \mathrm{~min}\right.$ ). An interaction between the drug and polymer was revealed in FTIR studies. No drug crystals were seen in SEM studies indicating its presence in a substantially amorphous state in the SD. The statistical analysis of in vitro dissolution data of screening study revealed that high viscosity hypromellose and diluent type had significant effect $(p<0.05)$ on dissolution while the hardness of the tablet had no significant effect $(p>0.05)$. The mixture design thereafter predicted the optimum formula through a significant model. High and low viscosity hypromellose (66:44) at $\sim 17 \% \mathrm{w} / \mathrm{w}$ of matrix provided the desired drug release control. Conclusion: Dissolution enhancement could be attained by SD prepared using solvent free process. D-optimal mixture design approach, where the unit weight remains constant in spite of controlled variation in level of factors studied, was found to be a promising approach in pharmaceutical formulation optimization. A combined approach of SD and CR was found to be a promising strategy for better control of the drug release in a relatively simple monolayer matrix compared to a complex trilayer design.
\end{abstract}

Key words: Nisoldipine, Solid Dispersion, Design of Experiments, Screening, Mixture Design

\section{INTRODUCTION}

Hydrophillic matrices are one of the most popular approaches for drug release control. However, for poorly soluble drugs like NIS, such systems are inefficient and might lead to incomplete drug release. ${ }^{1}$ This means that a portion of the drug could be retained in the polymeric matrix and is not released completely. Recently, there has been an advent of use of new drug delivery approaches to address the poor solubility and potential bioavailability issues. ${ }^{2}$ Often complex technologies are being used to address these
Submission Date: 19-01-2017; Revision Date: 02-03-2017; Accepted Date: 30-03-2017

DOI: 10.5530/ijper.51.2s.54 Correspondence: Sandeep kumar,

Product Development and Research, Sun Pharmaceutical Industries Limited, Sector-18, Gurgaon-122015, INDIA. Tel.no: 0124-4194530

E-mail: msk.vats@gmail.com

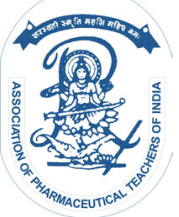

www.ijper.org 
issues, as in Sular ${ }^{\mathrm{TM}}$, the marketed product of NIS, where a trilayer matrix tablet design (Geomatrix ${ }^{\mathrm{TM}}$ technology) consisting of a hydrophilic matrix core of NIS and one or two impermeable or semi-permeable polymeric coatings (films or compressed barriers) are applied on one or both bases of the core. ${ }^{3}$ However, trilayer tablets are difficult to manufacture and scale-up using the conventional press technology. Also, poorly soluble drugs when incorporated into extended release (ER) systems can further aggravate the variability in the pharmacokinetics and bioavailability. Since the bioavailability of NIS is dissolution rate limited (BCS class-II), an approach to enhance solubility is required. Incorporation of a solubility enhanced form like solid dispersion into an ER module can significantly mitigate the problem of incomplete release and help provide desired drug release control. An initial quality risk assessment (IQRA) approach is advocated by global regulatory agencies to study the impact of factors affecting the quality attributes in drug product design. ${ }^{4}$ These guidelines emphasize on the use of quality by design (Qbd) and design of experiments (DoE) tools in formulation development. A common limitation in conventional design of experiments for matrix tablets is the variation is formulation weight due to controlled variation in factors under investigation. However, for controlled release matrix systems, the unit weight and surface area significantly impact the drug release kinetics. ${ }^{5} \mathrm{~A}$ mixture design of experiments ensures that the unit weight remains constant in all batches. ${ }^{6}$ To address the issues associated with ER formulation development of a poorly soluble drug, the present research work, proposes to enhance the solubility of BCS class -II drug using solid dispersion approach followed by use of D-optimal mixture design for optimization of the unit composition.

\section{MATERIALS AND METHODS}

\section{Materials}

NIS was procured from Erregierre, Italy. Copovidone (Kollidon VA-64) was obtained from BASF Corporation, USA. Hypromellose grades (K100MCR, K4 MCR) were obtained from Colorcon, India. Mannitol (Pearlitol SD 200) was sourced from Roquette, USA and Lactose (DCL-11) was obtained from DFE Pharma, USA. Microcystalline cellulose (Avicel 101) was obtained from FMC biopolymers, USA. Magnesium stearate and Aerosil-200 were sourced from Macron Inc., USA and Evonik, India respectively. Various ingredients used in the study were of analytical/pharmaceutical grade. NIS is prone to photolytic degradation and hence all the experiments were carried out using golden fluorescent light and analysis was carried out using low actinic amber color glassware.

\section{METHODS}

\section{Phase solubility study}

Solubility measurements were performed in triplicate using the method reported by Higuchi and Connors. ${ }^{7}$ An excess amount of NIS was added to purified water containing increasing concentrations $(0-10 \% \mathrm{w} / \mathrm{v})$ of copovidone. The vials were sealed and shaken at $37 \pm 0.5^{\circ}$ for $72 \mathrm{~h}$ in a thermostatically controlled orbital shakercum incubator (Colton, India) and the samples were filtered through a $0.45 \mu$ polyvinylidene fluoride (PVDF) filter. The filtrate was suitably diluted and the concentration in the solution was determined spectrophotometrically at $\lambda \max 238 \mathrm{~nm}$ (UV-2450, Shimadzu, Japan).

\section{Preparation of solid dispersion by hot melt extrusion}

For solid dispersions preparation using the hot melt extrusion technique, ${ }^{8}$ the drug and polymer were mixed geometrically and the blend was fed into extrusion chamber of a co-rotating twin screw hot-melt extruder (Pharm11, Thermo Fisher Scientific, Germany) at a constant feed rate of $50 \mathrm{rpm}$ and a powder feed rate of $200 \mathrm{~g} / \mathrm{h}$. The length to diameter ratio was 40 and the temperature of seven heating zones starting from hopper side towards the die ( $2 \mathrm{~mm}$ round) were set at $110,130,150,150,170,180$ and $180^{\circ} \mathrm{C}$. The extruded thread was subsequently passed through an in-line milling unit. The milled extrudes were cooled to room temperature and pulverized and screened via $425 \mu$ sieve (\#40 ASTM).

\section{Infrared spectral analysis}

Pure drug and solid dispersions were characterized by FTIR (Pristige-21, Shimadzu-Japan) spectroscopic analysis to study the drug and carrier interaction. A finely ground, approximately $1 \%$ mixture of a solid sample in $\mathrm{KBr}$ was fused into a transparent disk using a hydraulic press and analyzed over the range of 4000 to $400 \mathrm{~cm}-1$.

\section{Scanning electron microscopy}

The solid dispersion was screened by using scanning electron microscopy (Jeol-JSM-5300, Japan). The powder sample was suitably mounted on a glass stub and coated under vacuum with gold in an argon atmosphere. The screening was performed to see presence of characteristic needle shaped crystals of the drug. 


\section{Manufacturing of controlled release tablets containing solid dispersion}

The solid dispersion powder and excipients, other than lubricants, were screened via \#30 ASTM and mixed to form a blend. Magnesium stearate and aerosil-200 mixture was screened via \# 60ASTM. The blend and lubricants were mixed in a $500 \mathrm{ml}$ empty HDPE bottle attached to a blending shaft for 10 minutes (batch size500 tablets). The blends were compressed into tablets on a 16-station rotary (Cadmach, India) using two standard round concave; $8 \mathrm{~mm}$ punch and die sets.

\section{Dissolution studies}

The in-vitro dissolution behavior of matrix tablets containing solid dispersion were studied using dissolution system (2100C, Distek Inc., USA) equipped with autosampler (Evolution 4300, Distek Inc., USA). The dissolution studies for tablets containing (17 $\mathrm{mg}$ dose) were performed using USP Dissolution apparatus type II (paddle) in $0.1 \mathrm{~N} \mathrm{HCl}$ containing $0.25 \%$ SLS (900 ml) dissolution media at $50 \mathrm{rpm}$ and $37 \pm 0.5^{\circ} \mathrm{C}$ temperature $(\mathrm{n}=6)$. The dissolution test was performed for $12 \mathrm{~h}$ with $5 \mathrm{ml}$ sampling and replacements with same volume of fresh media post each sampling. The samples were filtered using $0.45 \mu$ PVDF filter, diluted and analyzed by UV spectrophotometer at $238 \mathrm{~nm}$. Cumulative amount of drug dissolved (with sampled volume adjustment) was calculated using a linear calibration equation, over range of $1-20 \mu \mathrm{g} / \mathrm{ml}$.

\section{Design of experiments and statistical analysis}

JMP ${ }^{\circledR}$ software (version 12, SAS Inc., USA) was used for the evaluation of the statistical experimental design. Means were compared by analysis of variance (ANOVA). The level of significance was set at $\alpha=0.05$. Suitable regression models were generated and response surface methodology was used to search for an optimized formula.

\section{Evaluation of drug release kinetics}

The in vitro drug release data of the optimized formulation was analyzed with various mathematical models $^{10}$ to ascertain the drug release kinetics using DD-Solver, an MS-Excel add-in software package. ${ }^{10,11}$

\section{RESULTS AND DISCUSSION}

\section{Phase solubility study}

The aqueous solubility of NIS, a BCS class-II drug was determined to be $5.913 \mu \mathrm{g} / \mathrm{ml}$. The saturation solubility of drug was evaluated in copovidone solutions at $0-10 \%$ $\mathrm{w} / \mathrm{w}$ concentration. The phase solubility curve is shown in Figure 1.
The solubility of NIS increased as a function of polymer concentration. The data was modeled into a linear trend line $\left(y=20.947+6.6696, r^{2}=0.9869\right)$ and it followed an $A_{L}$-type phase solubility curve. ${ }^{12}$ The drastic increase in solubility with the increased copovidone concentration can be reasoned basis chemistry of the polymer. The combination of $40 \%$ by weight of vinyl acetate content and $60 \%$ of poly vinyl pyrrolidone (40:60 ratio) might be the reason for the better drug-polymer interaction facilitating solubilization.

\section{Infrared spectral analysis}

In order to study the type of interaction between the drug and polymer which made abrupt increase in solubility and dissolution, FTIR studies were conducted Figure 2. A sharp absorption band at $3321 \mathrm{~cm}^{-1}$ was observed for pure NIS due to stretching of N-H group of the dihydropyrimidine (DHP) moiety in the drug substance. Other characteristics bands were observed at $2967 \mathrm{~cm}^{-1}\left(\mathrm{C}_{\mathrm{sp} 3}-\mathrm{H}\right.$ stretching), $3102 \mathrm{~cm}^{-1}\left(\mathrm{C}_{\mathrm{sp} 2}-\mathrm{H}\right.$ stretching), 1656 and $1706 \mathrm{~cm}^{-1}(\mathrm{C}=\mathrm{N}$ and $\mathrm{C}=\mathrm{O}$ stretching $)$. A peak broadening was seen in the spectra of solid dispersion with copovidone corresponding to the stretching of drug-NH region. The observations were analogous to that reported in literature. ${ }^{13}$ This can be attributed to the hydrogen boding between $\mathrm{N}-\mathrm{H}$ group of the dihydropyrimidine moiety of the drug and the amide function / ester function of the copovidone polymer.

\section{Scanning electron microscopy}

The SEM showed sharp edged particles of solid dispersion owing to the glassy nature of the melt cooled polymer matrix. However, the characteristic needle shaped crystals of the drug were absent indicating that the solid dispersion was molecularly dispersed in the polymeric matrix Figure 3.

\section{D-optimal Screening design of experiments}

Hypromellose (HPMC) based hydrophilic matrices using a combination of low viscosity (type 2910, USP) and high viscosity (type 2208, USP) grades, are widely used in pharmaceutical industry for controlled release applications. ${ }^{14}$ Based on preliminary experiments, a directly compressible matrix was selected to formulate solid dispersion tablets for controlled drug release. The drug-copovidone solid dispersion at 20\% drug load was used. To form a simple monolayer matrix, a hypromellose based hydrophilic matrix was selected with a combination of high (HPMC K100M) and low (HPMC K4M) viscosity grades. The rationale for selection of combination of viscosity polymers was to fabricate a matrix system which can hydrate quickly due to low 


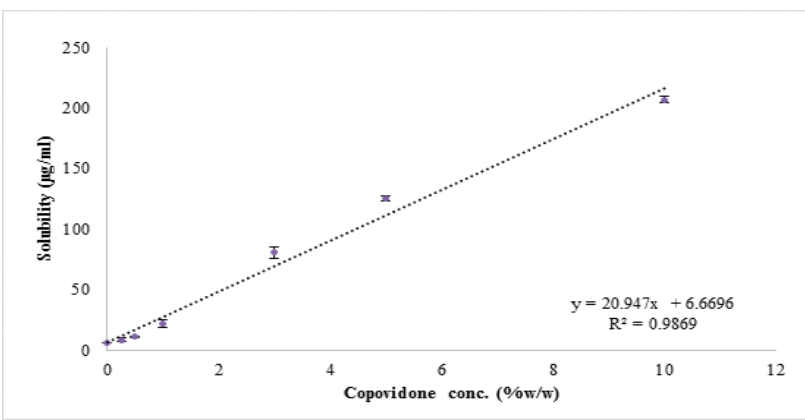

Figure 1: Phase solubility study of Nisoldipine in aqueous Copovidone phase (0-10\%).

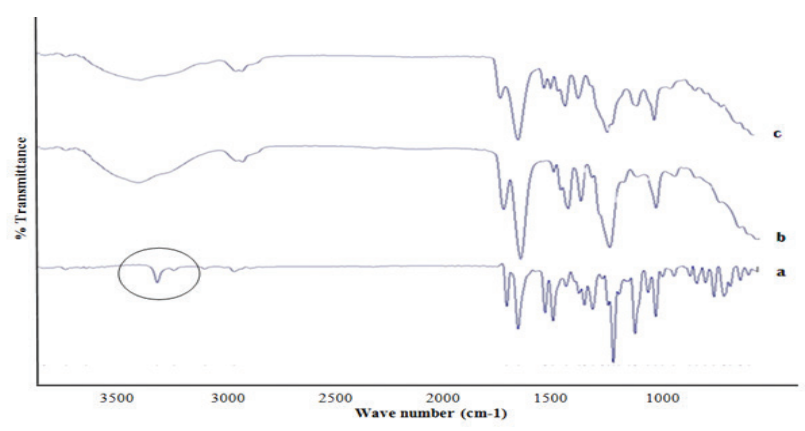

Figure 2: FTIR spectra of a. Nisoldipine b. Copovidone c. Solid dispersion with Copovidone ( $20 \%$ drug).

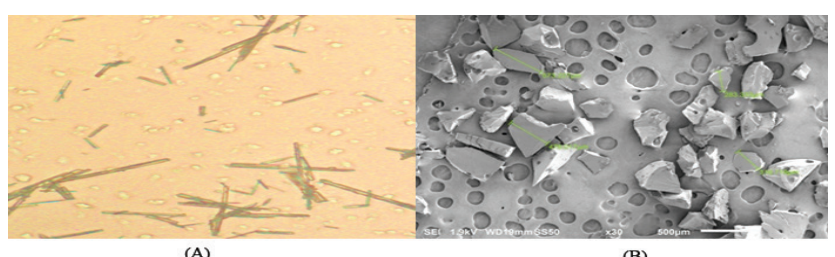

(B)

Figure 3: (A) Microscopy of Nisoldipine (B) SEM of Nisoldipine-copovidone solid dispersion

viscosity polymer and then sustain the release due to slow erosion of the high viscosity polymer. ${ }^{15} \mathrm{~A}$ risk assessment based approach was used to understand the factors affecting the drug release from such matrices. The high and low viscosity hypromellose were studied at two levels as continuous variables. The hydrophilic affinity of diluent also plays an important role in the rate of matrix hydration and hence three different type of diluents were studied as categorical variables. ${ }^{16}$ The drug release from matrix tablets is often governed by the surface area of the tablet which depends on tablet thickness. The tablet thickness, in turn, is affected by the hardness and hence a low and high degree of hardness (which directly affects tablet thickness) was also taken as a categorical variable for screening design. ${ }^{17}$ The variables and their levels studied along with experimental design and the responses are presented in Table 1.
The tablets complied with the pharmacopeial requirements of weight variation and friability. The selection of a D-optimal design was justified by the fact that a continuous variation in weight of the matrix tablet might result in different surface area of the tablets and might act as a key variable to affect the responses. A matrix design enables the variation in the levels of interest in such a way that the tablet weight remains the same. The statistical analysis of the data was carried out by standard least square method. The results of ANOVA analysis and model coefficients are shown in Table 2.

The model statistics indicates that the models generated were significant $(\mathrm{p}<0.05)$. Among the Hypromellose grades, high viscosity polymer was found to be the most significant term throughout the dissolution profile. Increasing the concentration of HPMC K100MCR in the tablet resulted in decrease in drug release. Among the three diluents studied, microcrystalline cellulose MCC was found to be a significant factor having a negative effect on the dissolution profile. This can be attributed primarily due to the fine particle size and water insoluble nature of this material. Mannitol based tablets; on the other hand, showed increase in dissolution as the level was increased. A co-processed excipient containing both mannitol and MCC have also been reported in literature. ${ }^{18}$

The diluents screened have different compressibility and had the potential to impact the tablet hardness. Interestingly, the hardness of tablet was not found to be a significant factor affecting the drug release. This might be attributed to the presentation of poorly soluble drug in a solid dispersion system and then incorporating it into an extended release dosage form. This is one key advantages of the current research and thus the system is proposed to be a robust and simple system compared to the other ER dosage forms of BCS class II drug. The mixture design attributed to maintain a similar surface area across the formulations.

Based on the prediction from model generated through design of experiments, a batch was manufactured to confirm the predicted drug release profile (formulation code OP1), the composition of which is provided in Table 3. The tablets complied with the pharmacopeial requirements of weight variation and friability.

The actual versus predicted profile of the final formulation showed an $\mathrm{F}_{2}$ value (similarity factor) of 71 indicating a good correlation between the actual and predicted profile. The drug release profile was meeting the extended release criteria set as constraints for the target design and not less than $80 \%$ drug release in $12 \mathrm{~h}$ Table 1 . The 


\begin{tabular}{|c|c|c|c|c|c|c|c|c|}
\hline \multicolumn{5}{|c|}{ Factors } & Role & Level 1 & \multicolumn{2}{|c|}{ Level 2} \\
\hline \multicolumn{5}{|c|}{ Hypromellose K100MCR (X1) } & Continuous & 15 & \multicolumn{2}{|c|}{35} \\
\hline \multicolumn{5}{|c|}{ Hypromellose K4MCR (X2) } & Continuous & 10 & \multicolumn{2}{|c|}{15} \\
\hline \multicolumn{5}{|c|}{ Type of diluent / filler (X3) } & Categorical & Lactose & MCC & Mannitol \\
\hline \multicolumn{5}{|c|}{ Tablet hardness (X4) } & Categorical & Low $(4-6 \mathrm{kp})$ & \multicolumn{2}{|c|}{ High (8-10 kp) } \\
\hline \multicolumn{5}{|c|}{ Responses } & \multicolumn{4}{|c|}{ Constraints } \\
\hline \multicolumn{5}{|c|}{$\%$ Cumulative drug release at $2 \mathrm{~h}(\mathrm{Y} 1)$} & \multicolumn{4}{|c|}{$0 \% \leq \mathrm{Y} 1 \leq 30 \%$} \\
\hline \multicolumn{5}{|c|}{$\%$ Cumulative drug release at $4 \mathrm{~h}(\mathrm{Y} 2)$} & \multicolumn{4}{|c|}{$40 \% \leq Y 2 \leq 60 \%$} \\
\hline \multicolumn{5}{|c|}{$\%$ Cumulative drug release at $8 \mathrm{~h}(\mathrm{Y} 3)$} & \multicolumn{4}{|c|}{$80 \% \leq Y 3 \leq 100 \%$} \\
\hline \multicolumn{5}{|c|}{$\%$ Cumulative drug release at $12 \mathrm{~h}(\mathrm{Y} 4)$} & \multicolumn{4}{|c|}{ Maximize $(Y 4>80 \%)$} \\
\hline \multicolumn{5}{|c|}{ Experimental design } & \multicolumn{4}{|c|}{ Responses } \\
\hline \multirow[t]{2}{*}{ Code } & \multicolumn{4}{|c|}{ Variables } & \multicolumn{4}{|c|}{ ( $\%$ Cumulative drug release mean $\pm S D, n=3$ ) } \\
\hline & $\mathrm{X} 1$ & $\mathrm{X} 2$ & $\mathbf{X 3}$ & $\mathrm{X} 4$ & Y1 (2 h) & Y2 (4 h) & Y3 (8 h) & Y4 (12 h) \\
\hline M1 & 15 & 15 & $\mathrm{MCC}$ & High & $5 \pm 3.77$ & $16 \pm 1.68$ & $40 \pm 3.32$ & $66 \pm 3.35$ \\
\hline M2 & 15 & 15 & Lactose & Low & $25 \pm 1.83$ & $45 \pm 2.34$ & $88 \pm 2.37$ & $92 \pm 3.39$ \\
\hline M3 & 35 & 10 & $\mathrm{MCC}$ & Low & $3 \pm 0.58$ & $12 \pm 3.13$ & $31 \pm 4.25$ & $54 \pm 2.45$ \\
\hline M4 & 35 & 15 & Mannitol & Low & $6 \pm 2.08$ & $17 \pm 1.63$ & $36 \pm 3.21$ & $53 \pm 2.15$ \\
\hline M5 & 35 & 15 & Mannitol & High & $6 \pm 3.37$ & $18 \pm 3.26$ & $45 \pm 2.49$ & $67 \pm 3.36$ \\
\hline M6 & 35 & 10 & $\mathrm{MCC}$ & High & $3 \pm 3.06$ & $12 \pm 2.16$ & $31 \pm 3.35$ & $54 \pm 1.43$ \\
\hline M7 & 15 & 10 & Lactose & High & $25 \pm 2.52$ & $53 \pm 0.97$ & $88 \pm 4.12$ & $97 \pm 4.47$ \\
\hline M8 & 15 & 10 & Mannitol & High & $20 \pm 2.54$ & $47 \pm 1.24$ & $80 \pm 4.45$ & $88 \pm 4.38$ \\
\hline M9 & 35 & 15 & Lactose & High & $10 \pm 2.67$ & $25 \pm 1.48$ & $49 \pm 4.36$ & $75 \pm 4.56$ \\
\hline M10 & 35 & 10 & Lactose & Low & $8 \pm 4.59$ & $19 \pm 2.12$ & $45 \pm 3.36$ & $67 \pm 3.19$ \\
\hline M11 & 15 & 15 & MCC & Low & $5 \pm 0.87$ & $16 \pm 2.44$ & $40 \pm 2.26$ & $66 \pm 3.32$ \\
\hline M12 & 15 & 10 & Mannitol & Low & $17 \pm 1.14$ & $43 \pm 1.35$ & $76 \pm 1.57$ & $89 \pm 5.11$ \\
\hline
\end{tabular}

MCC- Microcrystalline cellulose,

\section{Table 2: Regression coefficients and level of significance for each response}

\begin{tabular}{|c|c|c|c|c|c|c|c|c|}
\hline \multirow{2}{*}{ Coefficients } & \multicolumn{2}{|c|}{ Y1 (2h) } & \multicolumn{2}{|c|}{ Y2 (4h) } & \multicolumn{2}{|c|}{ Y3 (8h) } & \multicolumn{2}{|c|}{ Y4 (12h) } \\
\hline & Coeff. & $p$-value & Coeff. & $p$-value & Coeff. & $p$-value & Coeff. & $p$-value \\
\hline Intercept & 11.13 & $<0.0001$ & 26.87 & $<0.0001$ & 54.15 & $<0.0001$ & 72.57 & $<0.0001$ \\
\hline K100 MCR $(15,35)$ & -5.03 & $0.0038^{*}$ & -9.87 & $0.0008^{*}$ & -14.67 & $0.0009^{*}$ & -10.58 & $0.0004^{*}$ \\
\hline K4 MCR $(10,15)$ & -1.64 & 0.1873 & -4.05 & $0.0450^{*}$ & -4.36 & 0.1190 & -2.6 & 0.1292 \\
\hline Diluent [MCC] & -6.96 & $0.0042^{*}$ & -12.79 & $0.0013^{*}$ & -18.58 & $0.0015^{*}$ & -12.44 & $0.0010^{*}$ \\
\hline Diluent [Mannitol] & 1.03 & 0.5309 & 4.25 & 0.1103 & 5.16 & 0.1785 & 1.98 & 0.3809 \\
\hline Hardness [Low] & -0.53 & 0.6442 & -1.60 & 0.3604 & -1.35 & 0.5940 & -2.12 & 0.2014 \\
\hline \multicolumn{9}{|c|}{ Model statistics } \\
\hline $\mathrm{R}^{2}$ & \multicolumn{2}{|c|}{0.89} & \multicolumn{2}{|c|}{0.93} & \multicolumn{2}{|c|}{0.92} & \multicolumn{2}{|c|}{0.94} \\
\hline Adj. $R^{2}$ & \multicolumn{2}{|c|}{0.88} & \multicolumn{2}{|c|}{0.91} & \multicolumn{2}{|c|}{0.91} & \multicolumn{2}{|c|}{0.92} \\
\hline Prob. $>\mathrm{F}$ & \multicolumn{2}{|c|}{0.054} & \multicolumn{2}{|c|}{0.012} & \multicolumn{2}{|c|}{0.0087} & \multicolumn{2}{|c|}{0.0095} \\
\hline
\end{tabular}

$p$-value is Prob $>|t|, *$ indicates $p<0.05$, significant term 
Table 3: Final formula and drug release kintics of monolayer matrix tablets

\begin{tabular}{|c|c|c|}
\hline \multicolumn{3}{|c|}{ Composition [OP1] } \\
\hline \multicolumn{2}{|c|}{ Ingredients } & $m g / t a b$ \\
\hline \multicolumn{2}{|c|}{ Solid eqvt. to $17 \mathrm{mg}$ dose } & 85.00 \\
\hline \multicolumn{2}{|c|}{ Hypromellose K100 MCR } & 28.20 \\
\hline \multicolumn{2}{|c|}{ Hypromellose K4MCR } & 14.45 \\
\hline \multicolumn{2}{|c|}{ Mannitol } & 120.75 \\
\hline \multicolumn{2}{|c|}{ Magnesium stearate } & 0.50 \\
\hline \multicolumn{2}{|c|}{ Aerosil 200} & 1.50 \\
\hline \multicolumn{2}{|c|}{ Total Tablet wt. } & 250.00 \\
\hline \multicolumn{3}{|c|}{ Drug Release kinetics } \\
\hline Korsmeyer-peppas & \multicolumn{2}{|c|}{$r^{2}=0.99, K_{K P}=7.550, A I C=6.89, n=0.93$} \\
\hline Higuchi & \multicolumn{2}{|c|}{$r^{2}=0.79, K_{H}=18.90, \quad A I C=18.75$} \\
\hline First-order & \multicolumn{2}{|c|}{$r^{2}=0.99, \quad K_{1}=0.097, \quad A I C=15.59$} \\
\hline Zero order & \multicolumn{2}{|c|}{$r^{2}=0.99, \quad K_{0}=6.44, \quad A I C=8.43$} \\
\hline \multicolumn{3}{|c|}{$\mathrm{AIC}=\mathrm{Akaike}$ information criteria } \\
\hline
\end{tabular}

drug content in optimized formulation was found to be $100.5 \pm 0.37 \%(\mathrm{n}=3)$.

To investigate the drug release mechanism from the matrix tablets, the drug release kinetics was studied using various release kinetics models. Among the various mathematical models studied, overall $\mathrm{r}^{2}$ and AIC value criteria were used to select the most appropriate model. ${ }^{19}$ The parameters obtained are listed in Table 3. The drug release kinetics follows a Korsmeyer-Peppas model based on the lowest AIC value criteria. The $n$ value indicates a non-fickian or anomalous diffusion pattern. This means that both the diffusion and erosion mechanisms were prevalent.

\section{CONCLUSION}

A scalable, solvent free approach of hot-melt extrusion was used to prepare dissolution enhanced solid dispersion for NIS. Contrary to the trilayer tablet drug delivery approach used in marketed product, a simple monolayer hydrophilic matrix system was designed through statistical design of experiments. Combination of high and low viscosity Hypromellose (66:44) at $\sim 17 \% \mathrm{w} / \mathrm{w}$ of matrix provided the desired drug release control of $12 \mathrm{~h}$. Presence of dissolution enhanced form of poorly soluble drug (SD) showed to offer an advantage of a matrix tablet free from effect of hardness, resolving probable high speed scale-up issues. A key challenge faced during optimization of tablets is change in weight and surface area due to factors under study being varied. D-optimal mixture design approach, where the unit weight remains constant in spite of controlled variation in level of factors studied, is a promising approach in pharmaceutical formulation optimization to address this problem. The drug release could be controlled as per the set constraints for desired extended release, providing flexibility of tailoring the drug release and it followed erosion and diffusion drug release kinetics.

The present research work has paved way for studying combination systems utilizing solubility enhanced and controlled release of poorly soluble drugs in the same drug delivery module, for a wide variety of therapeutic applications.

\section{ACKNOWLEDGEMENT}

Authors are highly thankful to SPIL, R\&D, Gurgaon for providing research facilities.

\section{CONFLICT OF INTEREST}

Authors have no conflict of interest with the content of this article.

\section{REFERENCES}

1. Janssens S, Van Den MG. Enhancing solubility and dissolution rate of poorly soluble drugs: United states patent application no. US 20110059175 A9, April 2007.

2. Park K. Controlled drug delivery systems: past forward and future back. J Control Rel. 2014;190:3-8. https://doi.org/10.1016/j.jconrel.2014.03.054; PMid:24794901 PMCid:PMC4142099.

3. Jain $\mathrm{D}$, Raturi $\mathrm{R}$, Jain $\mathrm{V}$, et al. Recent technologies in pulsatile drug delivery systems. Bio matter. 2011;1(1):57-65. https://doi.org/10.4161/ biom.1.1.17717; PMid:23507727 PMCid:PMC3548250.

4. $\mathrm{ICH}$ Harmonized Tripartite guideline- Qualiy risk management Q9. Current step 4. 2005

5. Siepmann J, Peppas N. Hydrophilic matrices for controlled drug delivery: an improved mathematical model to predict the resulting drug release kinetics (the "sequential layer" model). Pharm Res. 2000;17(10):1290-8. https://doi. org/10.1023/A:1026455822595; PMid:11145237. 
6. Bodea A, Leucuta SE. Optimization of hydrophilic matrix tablets using a D-optimal design. Int J pharm. 1997;153(2):247-55. https://doi.org/10.1016/ S0378-5173(97)00117-8

7. Higuchi T, Connors KA. Phase-solubility techniques. Adv Anal Chem Instrum. 1965;4:117-212.

8. Karanth H, Shenoy VS, Murthy RR. Industrially feasible alternative approaches in the manufacture of solid dispersions: A technical report. AAPS PharmSciTech. 2006;7(4):E31-8. https://doi.org/10.1208/pt070487; PMid:17233539 PMCid:PMC2750324.

9. Kim M-S, Kim J-S, You Y-H, et al. Development and optimization of a novel oral controlled delivery system for tamsulosin hydrochloride using response surface methodology. Int J Pharm. 2007;341(1):97-104. https://doi. org/10.1016/j.ijpharm.2007.03.051; PMid:17499949.

10. Costa P, Lobo JMS. Modeling and comparison of dissolution profiles. Eur J Pharm Sci. 2001;13(2):123-33. https://doi.org/10.1016/S09280987(01)00095-1.

11. Zhang $\mathrm{Y}$, Huo $\mathrm{M}$, Zhou J, et al. DDSolver: an add-in program for modeling and comparison of drug dissolution profiles. The AAPS J. 2010;12(3):263-71. https:// doi.org/10.1208/s12248-010-9185-1; PMid:20373062 PMCid:PMC2895453

12. Pralhad T, Rajendrakumar K. Study of freeze-dried quercetin-cyclodextrin binary systems by DSC, FT-IR, X-ray diffraction and SEM analysis. J pharm Biomed Anal. 2004;34(2):333-9. https://doi.org/10.1016/S07317085(03)00529-6.

\section{PICTORIAL ABSTRACT}

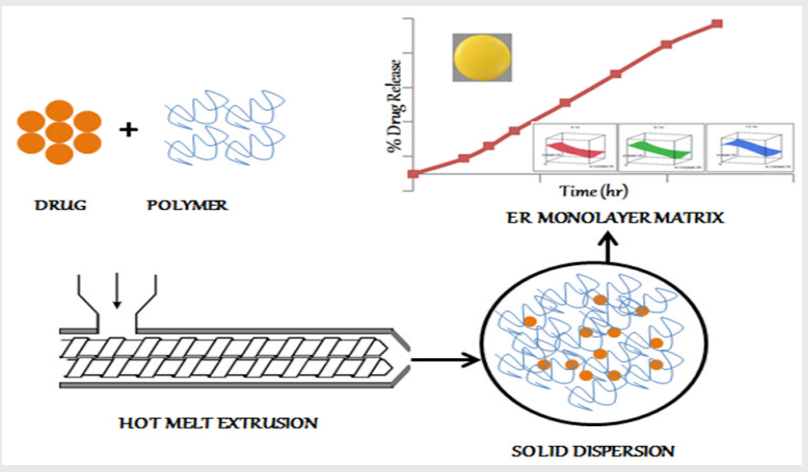

\section{About Authors}

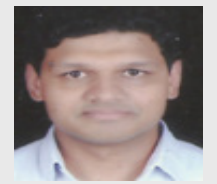

Sandeep Kumar: Sandeep Kumar is presently working as Senior Manager, Product Development at SPIL, Gurugram. His areas of interest include development, scale-up and technology transfer for oral solid dosage forms.

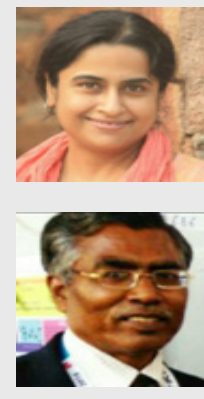

Dr Romi singh: Is Associate Vice President at SPIL, Gurugram. She has $\sim 20$ years of experience in pharmaceutical product development and drug delivery. Her area of interest encompasses formulation development on novel drug delivery platforms.

Prof. (Dr.) RN Gupta: Is a Professor, Pharmaceutical Sciences and Technology at BIT Ranchi. HE has more than 24 years of experience in the area of pharmaceutics. He has played instrumental role in the field of pharmacovigilance and drug regulation in India. His research interests include novel drug delivery systems for neglected diseases.

Cite this article: Kumar S, Singh R and Gupta RN. Optimization of monolayer hydrophilic matrix tablets containing Nisoldipine solid dispersion using D-optimal design. Indian $\mathrm{J}$ of Pharmaceutical Education and Research. 2017;51(2s):91-7. 